\title{
XMM-Newton observations of M 87 and its X-ray halo
}

H. Böhringer ${ }^{1}$, E. Belsole ${ }^{2}$, J. Kennea ${ }^{3}$, K. Matsushita ${ }^{1}$, S. Molendi ${ }^{4}$, D. M. Worrall ${ }^{5}$, R. F. Mushotzky ${ }^{6}$, M. Ehle ${ }^{7}$ M. Guainazzi ${ }^{7}$, I. Sakelliou ${ }^{8}$, G. Stewart ${ }^{9}$, W. T. Vestrand ${ }^{10}$, and S. Dos Santos ${ }^{9}$

1 Max-Planck-Institut für Extraterrestrische Physik, 85748 Garching, Germany

2 CEA Saclay, Service d'Astrophysique, 91191 Gif-sur-Yvette, France

3 Department of Physics, University of California, Santa Barbara, CA 93106, USA

4 Istituto di Fisica Cosmica, Via Bassini 15, 20133 Milano, Italy

5 Department of Physics, University of Bristol, Tyndall Avenue, Bristol BS8 1TL, UK

6 Laboratory for High Energy Astrophysics, Code 660, NASA/Goddard Space Flight Center, Greenbelt, MD 20771, USA

7 XMM-Newton SOC, VILSPA-ESA, PO Box/Apartado 50727, 28080 Madrid, Spain

8 Mullard Space Science Laboratory, University College London, Holmbury St Mary, Dorking, Surrey RH5 6NT, UK

9 Department of Physics and Astronomy, The University of Leicester, Leicester LE1 7RH, UK

10 NIS-2, MS D436, Los Alamos National Laboratory, USA

Received 2 October 2000 / Accepted 2 November 2000

\begin{abstract}
We report performance verification observations of the giant elliptical galaxy M 87 in the Virgo Cluster with the MOS, pn, and optical monitor instruments on board of XMM-Newton. With the energy sensitive imaging instruments MOS and pn we obtain the first spatially constrained X-ray spectra of the nucleus and the jet of the galaxy. The good photon statistics of the pn and MOS allow a detailed analysis of the radial temperature and abundance distribution of 6 elements. The data provide no indication of a multi-temperature structure for radii $\geq 2$. An apparent sharp metal abundance drop deduced for the regions inside this radius is probably due to resonant line scattering.
\end{abstract}

Key words. galaxies: individual: M 87 - galaxies: clusters: individual: Virgo - X-rays: galaxies - galaxies: active

\section{Introduction}

The giant elliptical galaxy M 87 as the central dominant galaxy of the northern part of the Virgo cluster (e.g. Binggeli et al. 1987) displays a large variety of very interesting astrophysical phenomena (see e.g. Röser \& Meisenheimer 1997). Because M 87 is so bright and close, 17-20 Mpc (Freedman et al. 1994; Tammann \& Federspiel 1997), these astrophysical processes can be studied in M 87 in more detail than in any other comparable object.

M 87 (Virgo A, 3C 274) is one of the first known extragalactic radio sources and its halo is the first extragalactic X-ray source to have been identified (Bolton \& Stanley 1948; Byram et al. 1966). The highly peaked surface brightness profile of the extended X-ray emission subsequently observed with EINSTEIN implied an X-ray gas cooling time considerably smaller than the Hubble time, leading to the suggestion that M 87 harbours a significant "cooling flow" with a mass condensation rate of $\sim 10 M_{\odot} \mathrm{yr}^{-1}$ (Stewart et al. 1984; Fabian et al. 1984). EINSTEIN Crystal Spectrometer and Solid

Send offprint requests to: $\mathrm{H}$. Böhringer
State Spectrometer observations of M 87 provided X-ray line data which lent further support to the existence of a multi-temperature plasma in the core of the M 87 halo, as expected for a cooling flow model (Canizares et al. 1979, 1982; Mushotzky \& Szymkowiak 1988).

M 87 also houses an active nucleus with a one-sided jet observed at radio (e.g. Owen 1989), optical (e.g. Sparks et al. 1996), and X-ray wavelengths (Schreier et al. 1982; Neumann et al. 1997; Harris et al. 1997, 1999). The power output of the jet and the unseen counterjet obviously feeds a complex system of inner $(r \leq 25 \mathrm{kpc})$ and outer radio lobes $(r \leq 40 \mathrm{kpc}$; e.g. Owen et al. 2000) which partly interact with the thermal gas giving rise to distinct features in the X-ray surface brightness distribution (e.g. Belsole et al. and references therein). The ability to obtain sensitive spatially resolved X-ray spectroscopy with the $X M M$-Newton observatory offers the opportunity to gain new insights into these phenomena.

This paper reports on the analysis of the $X M M$ Newton (Jansen et al. 2001) performance verification phase observation of M 87 including results from the MOS (Turner et al. 2001), pn (Strüder et al. 2001) and Optical 


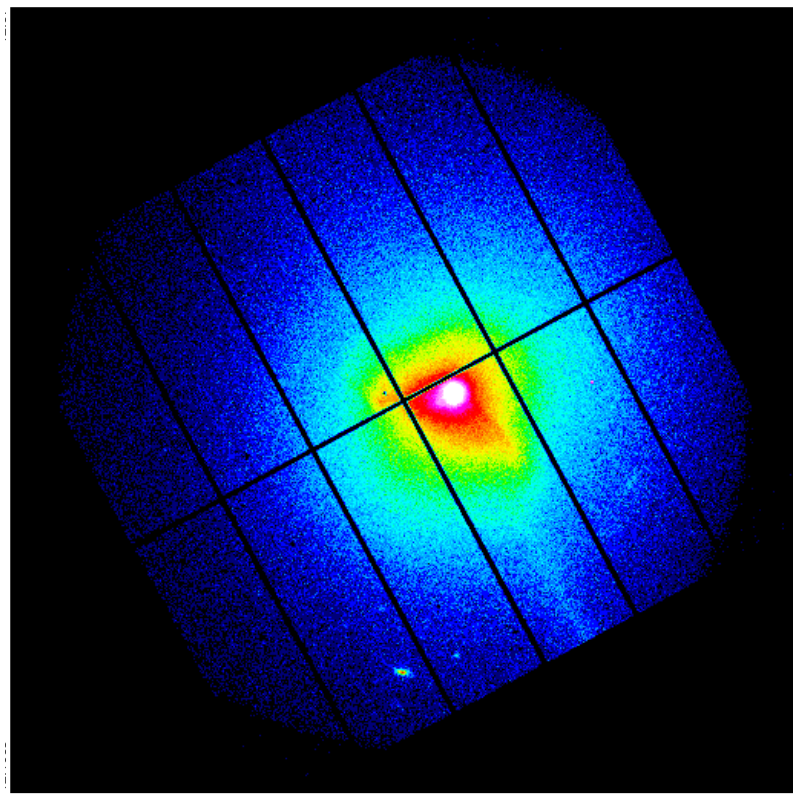

Fig. 1. XMM-Newton pn image of M 87 in the energy range 0.5 to $2 \mathrm{keV}$. The image size is about $26^{\prime}$ by $27^{\prime}$, north is up and east is left

Monitor (Mason et al. 2001) instruments. An analysis of the morphology and spectroscopy of the X-ray surface brightness enhancements related to the radio lobes of M 87 is presented in an accompanying paper by Belsole et al. (2001), and the results from high resolution spectroscopy with the RGS instrument are discussed in a forthcoming paper by Sakelliou et al. (2000).

\section{XMM observations}

M 87 was observed with $X M M$-Newton on June 19th, 2000 for $75.6 \mathrm{ksec}$. The effective exposure of the pn detector with the thin filter is $25.9 \mathrm{ksec}$, with no signes of enhanced background events. For the data reduction the SAS software was used as available in August 2000. Figure 1 shows a full frame image of the pn-detector observation in the energy range 0.5 to $2 \mathrm{keV}$. The effects of out-of-time events registered during the reading of the camera chips can be seen as a thin luminous band in the lower chip, fourth from the left. The X-ray halo has an almost spherically symmetric appearance, except for two localized surface brightness enhancements to the SW and $\mathrm{E}$ of the M 87 nucleus. These features coincide with the inner radio lobes. In total more than 12 Million photon events were registered by this detector. For the MOS1 and MOS2 detectors an effective observing time of $32 \mathrm{ksec}$ is obtained after excluding the first $7 \mathrm{ksec}$ due to enhanced background in the 10-12 keV band.

\section{The nucleus and jet of $M 87$}

M 87's nucleus and famous jet as seen with the Optical Monitor camera with the UWV2 filter is shown in Fig. 2. A cross section of the deconvolved surface brightness

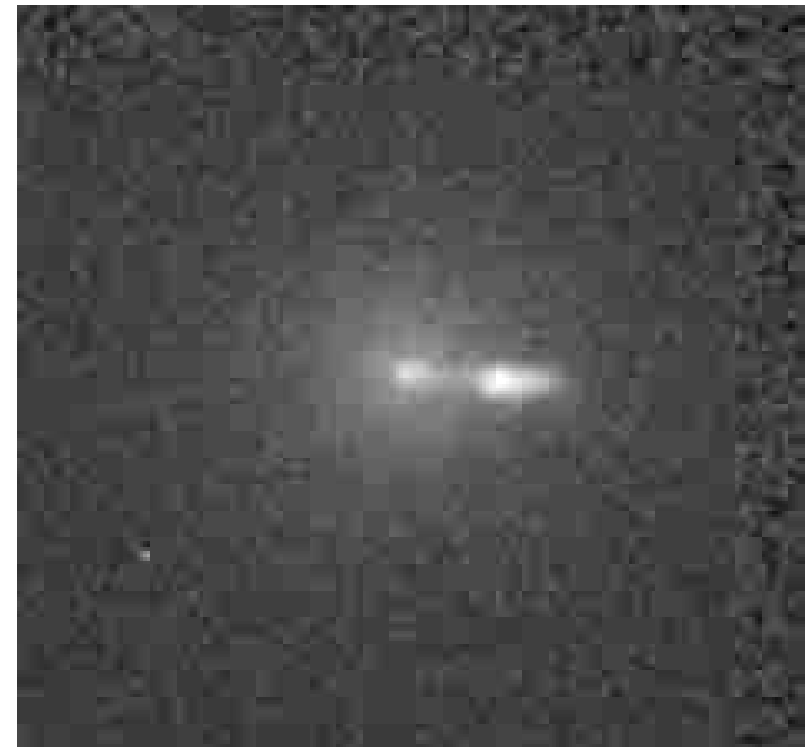

Fig. 2. UV image of the nucleus and the jet of $M 87$ observed with the optical monitor and the UVW2 filter $(\sim 180-230 \mathrm{~nm})$. North is not up in this image

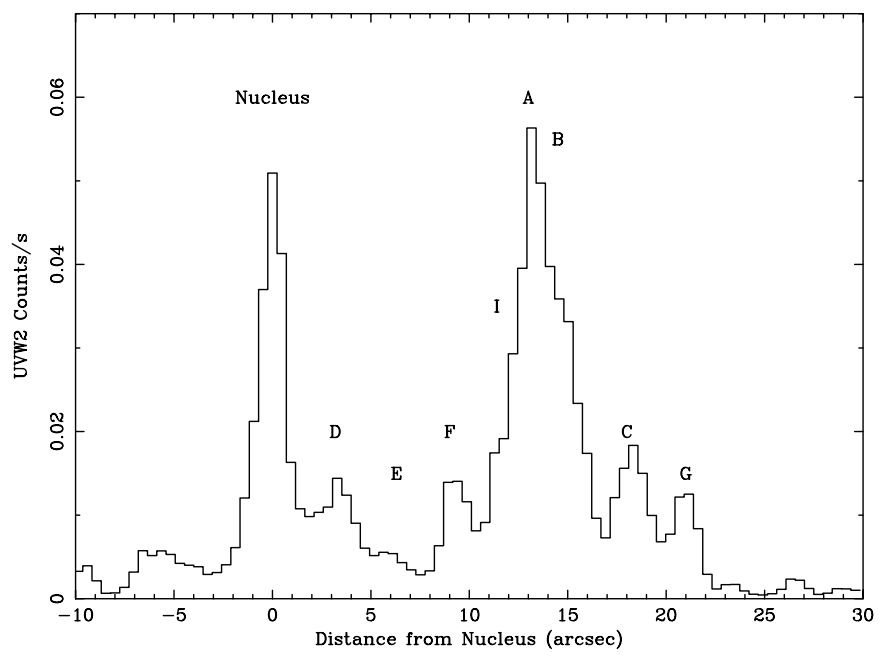

Fig. 3. Cross section through the deconvolved surface brightness of the UV image of the nuclear region in the line connecting the nucleus and the jet

distribution along the nucleus-jet direction is shown in Fig. 3. Several of the bright knots in the jet are easily visible. Interestingly, knot A in the jet is brighter than the nucleus (see Sparks et al. 1996). The observed count rate for the nucleus is $0.290 \mathrm{cts} \mathrm{s}^{-1}$ corresponding to a flux of $0.190 \pm 0.004 \mathrm{mJy}$ at $2120 \AA$.

Figure 4 shows the MOS2 X-ray image of the nuclear region. The nucleus and the jet appear as two point sources separated by about 11.5 arcsec (with a measurement uncertainty of about 0.5 arcsec). This is close to, but not exactly the same as, the nucleus to knot A separation of $13^{\prime \prime}$ seen in Fig. 3. This difference was already noted by Neumann et al. (1997) who quote a core-jet separation of 11.9 and 12.7 arcsec for the X-ray (ROSAT HRI) and radio image, respectively ( $1^{\prime \prime}$ corresponds to $82 \mathrm{pc}$ at the 


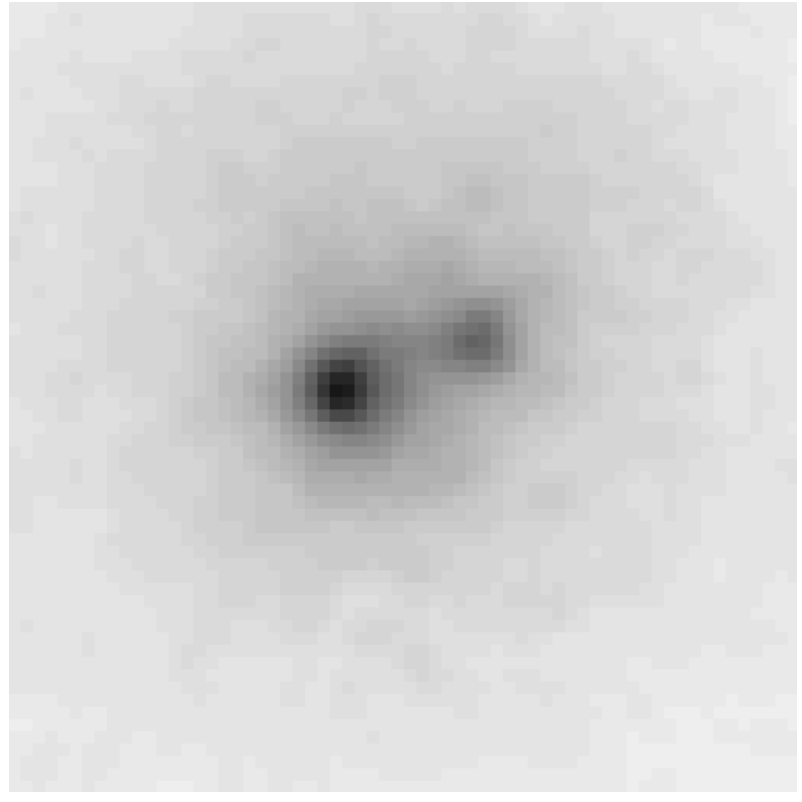

Fig. 4. MOS1 X-ray image image of the nucleus and the jet of M 87 in the energy range 0.3 to $5 \mathrm{keV}$. The side length of the image is 63 arcsec. The image was smoothed with a Gaussian filter with a $\sigma$ of 1 arcsec

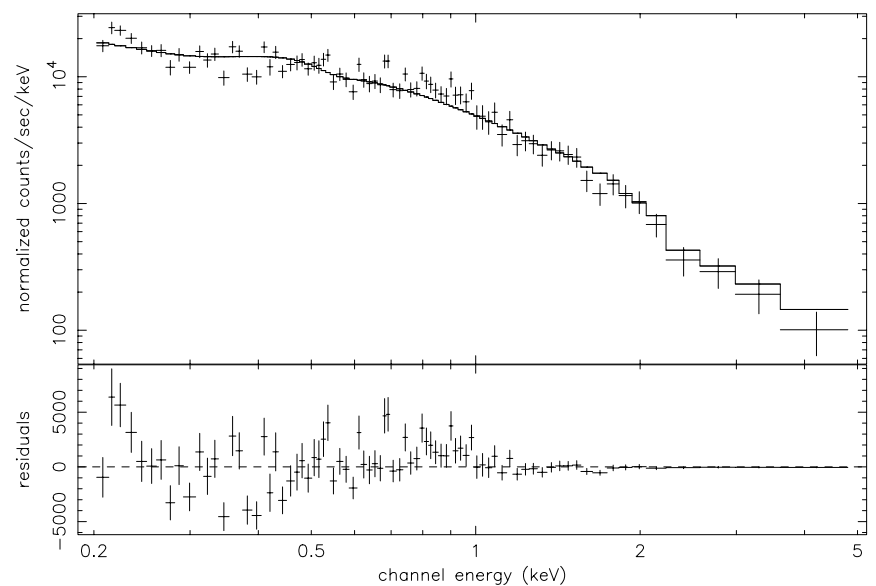

Fig. 5. XMM pn spectrum of the nucleus of M 87. The solid line shows a fit of an absorbed (galactic value) power law spectrum with a slope of 2.2

adopted distance to $\mathrm{M} 87$ of $17 \mathrm{Mpc})$. The $(2-10 \mathrm{keV})$ flux for the jet and the nucleus is about $5.510^{-13} \mathrm{erg} \mathrm{s}^{-1} \mathrm{~cm}^{-2}$ and $1.510^{-12} \mathrm{erg} \mathrm{s}^{-1} \mathrm{~cm}^{-2}$, respectively.

$X M M$-Newton allows us for the first time to obtain separate X-ray spectra for the nucleus and X-ray knot in the jet. For the analysis of the pn data we have extracted spectra from circular regions around each with a radius of 5 arcsec and taken two neighbouring regions of the same size for the background subtraction, one 20.8 arcsec in NNW and one 17.7 arcsec in SWW direction. The resulting spectra obtained using the first background region are shown in Figs. 5 and 6 . The spectra can be well fitted (in the 0.2 to $6 \mathrm{keV}$ band) by power law spectra with a slope of $2.2( \pm 0.2)$ for the nucleus and about $2.5( \pm 0.4)$ for the jet. There is no significant signature of thermal emission

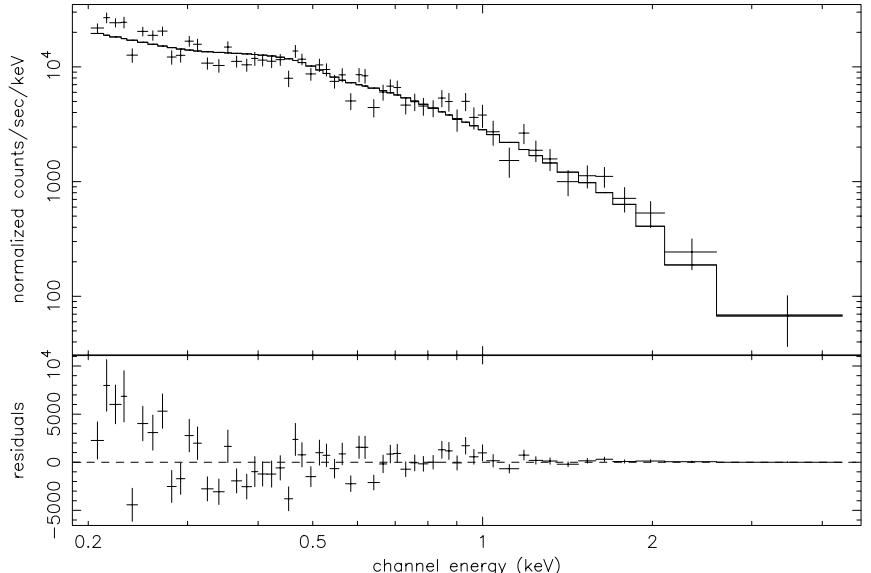

Fig. 6. XMM pn spectrum of the X-ray knot in the jet of M 87 . The solid line shows a fit of an absorbed (galactic value) power law spectrum with a slope of 2.5

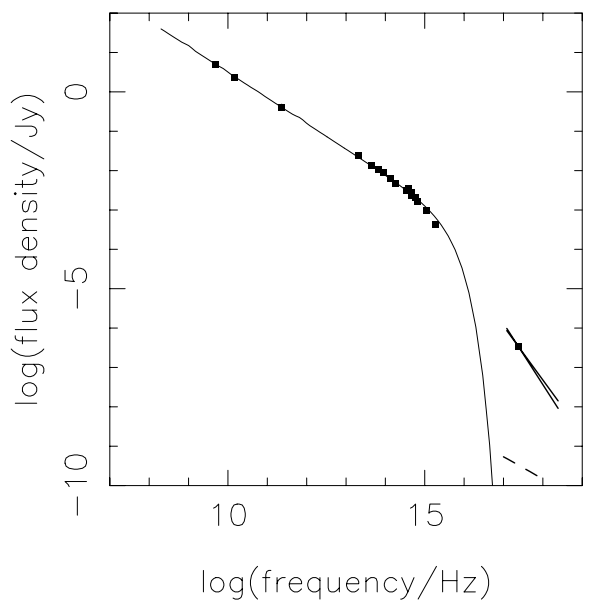

Fig. 7. Spectral energy distribution of knot A, B, and C of the jet in M 87 compared to a model of synchrotron emission (solid line) and self-Compton emission (broken line). The present observational result is marked by the data point and the two short lines indicating the lower and upper bound on the power law slope, respectively

(which can qualitatively be noted in Figs. 5 and 6 by an absence of a blended peak of iron lines around $1 \mathrm{keV}$ in the residual spectra).

Similar analysis was performed with the combined MOS data using an extraction radius of 4 arcsec for the nucleus and 3.5 arcsec for the jet and a background region at 22 arcsec distance from the nucleus. The results for the power low slope are $2.25\left[{ }_{-0.06}^{+0.04}\right]$ for the nucleus and $2.45\left[\begin{array}{l}+0.11 \\ -0.08\end{array}\right]$ for the jet (with acceptable fits with $\chi^{2}$ of $170 / 178$ d.o.f. and 101/110 d.o.f). A pure thermal origin of the jet emission can be ruled out with high confidence.

Figure 7 shows the radio to optical spectral energy distribution of the jet region encompasing knots $\mathrm{A}, \mathrm{B}$, and C (Biretta et al. 1991; Meisenheimer et al. 1996; Owen et al. 1989; Perola et al. 1980; Stocke et al. 1981; Tansley 2000) together with the $X M M-N$ ewton results. 


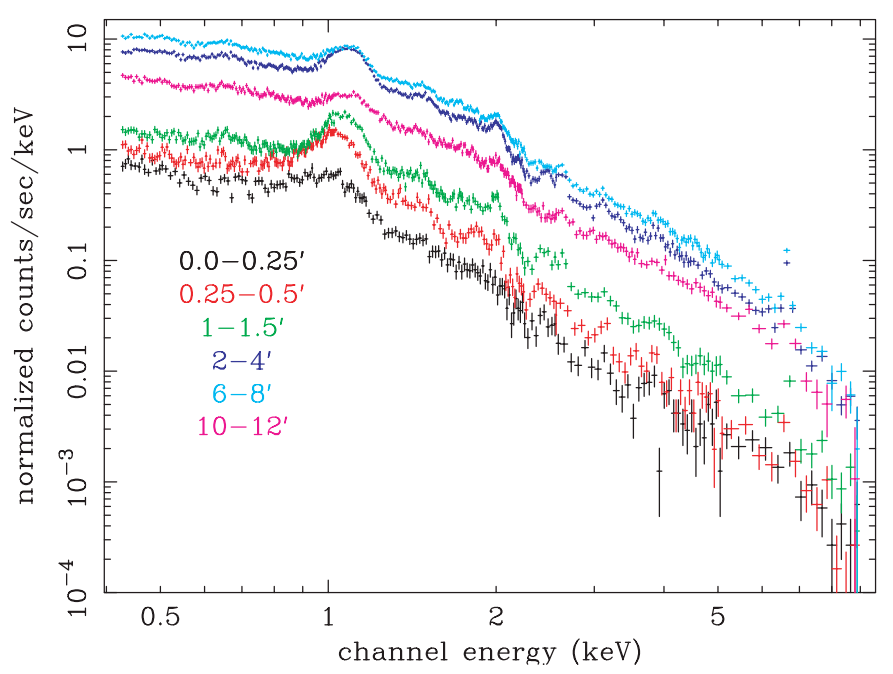

Fig. 8. pn spectra of the X-ray emission in concentric rings around M 87. Clearly seen is the variation of the emission line strength as a function of radius, indicating abbundance variations

\section{Temperature structure of the intracluster medium}

$X M M$-Newton allows us for the first time to analyse X-ray spectra in many regions of the M 87 X-ray halo with good photon statistics. Since the X-ray halo appears almost azimuthally symmetric, we study spectra in a set of concentric rings around the nucleus of $\mathrm{M} 87$. We exclude the regions of excess emission at the location of the radio lobes (a region of $4^{\prime}$ by $4^{\prime}$ centered on a point $2^{\prime}$ south and $2^{\prime}$ west and a region of $4.5^{\prime}$ by $4^{\prime} 2^{\prime}$ east and $0.5^{\prime}$ north of the nucleus, respectively) and the regions where the fraction of out of time events is large. We use the preliminary response matrix provided in July 2000 and an arf file calculated from vignetting data provided by B. Aschenbach (private communication). For the background, $70 \mathrm{ksec}$ of the Lockman hole observation in orbit 70, 71, and 73 cleaned of bright sources and background flares is used. A second analysis was performed with the cleaned MOS1 data. Vignetting corrected spectra were extracted from the event list. For the background a combination of several deep exposures collected by D. Lumb (private communication) where sources have been removed is used.

Resulting pn spectra are shown in Fig. 8. The fitting of model spectra was performed in XSPEC using the vmekal model. For all rings outside a radius of 1 arcmin twotemperature models provided no significant improvement over the fits of single-temperature models in the analysis of results of both detectors. For the inner circle a power law component was added to the fit. Figures 9 to 12 show the results of single temperature model fits for the pn and MOS1 data. Results of the different detectors agree well, except for some difference in the determination of the oxygen abundance (Fig. 12).

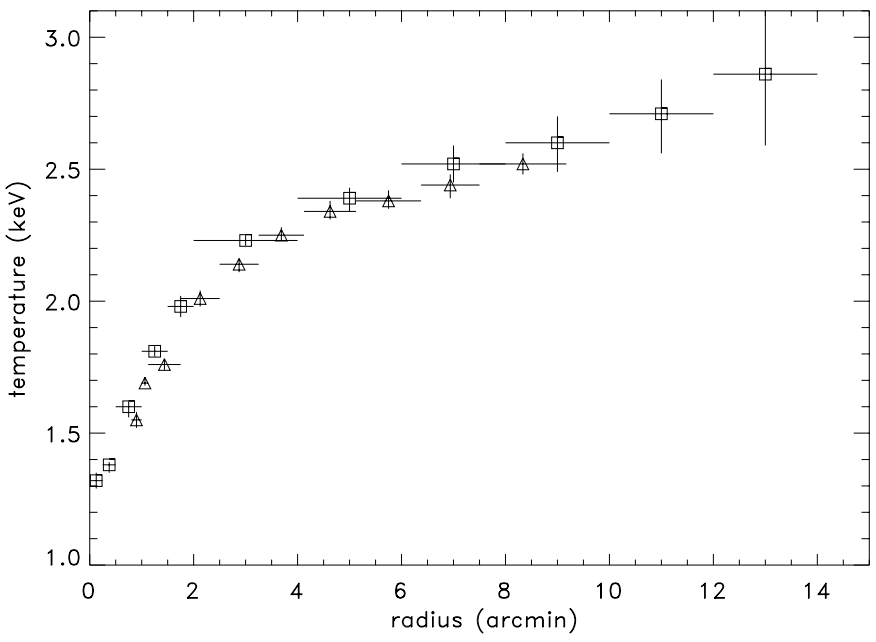

Fig. 9. Temperature profile determined from the MOS (triangles) and pn spectra (squares) in concentric rings around the M 87 nucleus excluding the regions of the X-ray lobes

The temperature profile shown in Fig. 9 provides a very tight constraint on the temperature distribution in the M 87 halo and it is in perfect agreement with the ASCA results from Matsumoto et al. (1996) and the BeppoSAX results of Guainazzi \& Molendi (2000). The temperatures are somewhat higher than determined with the ROSAT PSPC observation (Nulsen \& Böhringer 1995) but the trend with radius is similiar.

\section{Element abundances}

A comparison of the element abundances with the ASCA results of Matsumoto et al (1996) shows reasonable agreement for sulfur and the higher oxygen results obtained with the pn detector, while the ASCA results for silicon are on average about $30 \%$ and the iron abundances about 10-20\% lower. The equivalent width for the Si lines are in good agreement, however, and therefore the differences in the abundances may solely be due to the different plasma codes used (rs and vmekal).

In summary the profiles can be characterized by decreasing abundances with radius outside a radius of 1.5 arcmin, except for oxygen which is almost constant. Notable is the dramatic abundance decrease towards the center inside a radius of $1-1.5$ arcmin. We have further tested the reality of the decrease by considering twotemperature spectral models for the innermost three rings. For the best fitting models the upper temperature is only slightly higher than the temperature of the one-component fits and the lower temperature is roughly half this value. As shown in Fig. 10 for the examples of iron and silicon, the abundance decrease towards the center is smaller in the multi-component models than in the one-temperature fits, but it is still quite striking. 


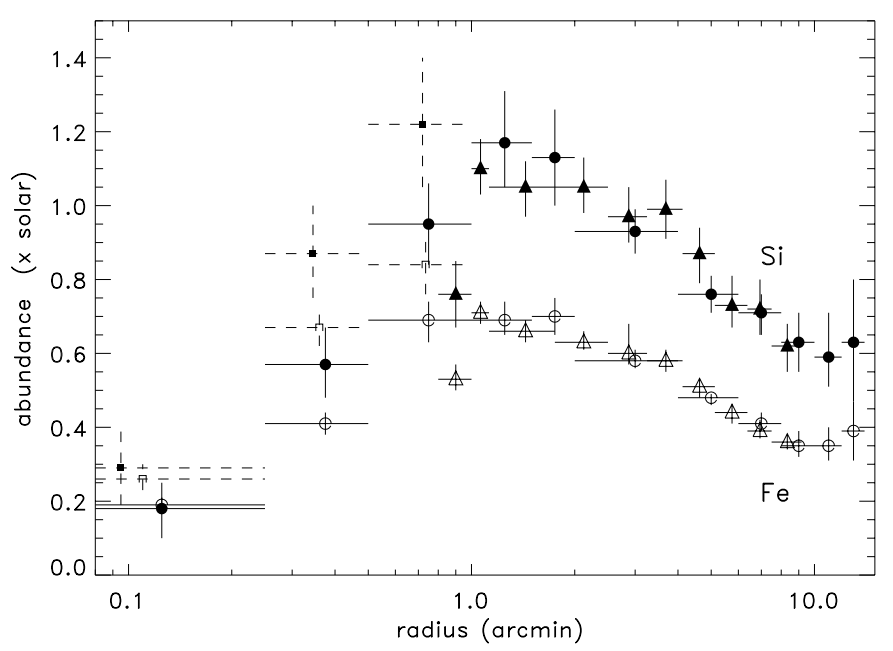

Fig. 10. Abundance profiles of Si (solid symbols) and Fe (open symbols) determined from the MOS1 (triangles) and pn spectra (circles) in concentric rings around the M 87 nucleus excluding the regions of the X-ray lobes. The data points with dashed error bars show the results of two-temperature fits to pn-spectra for $\mathrm{Si}$ (solid squares) and Fe (open squares)

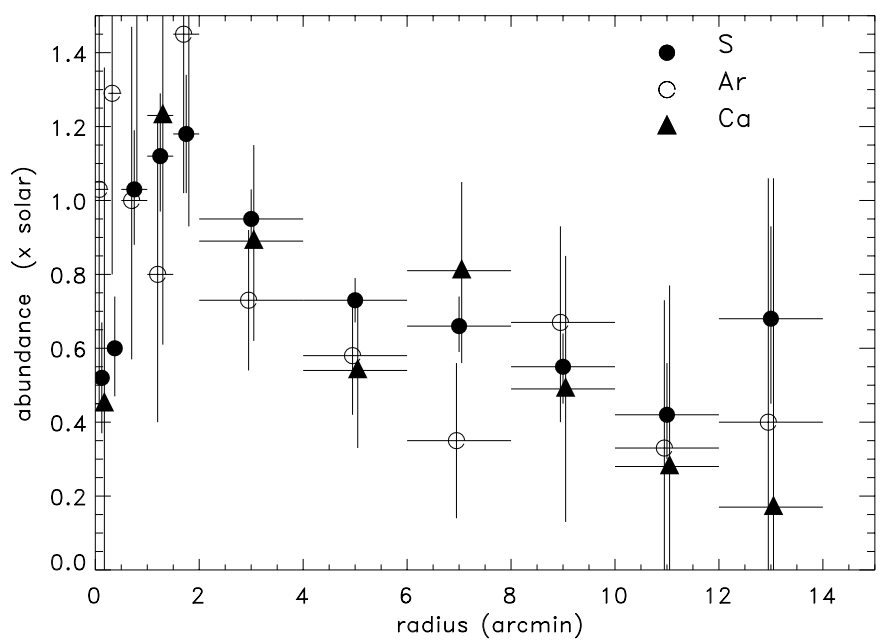

Fig. 11. Abundance profiles of S, Ar, Ca determined from pn spectra in concentric rings around the M 87 nucleus excluding the regions of the $\mathrm{X}$-ray lobes

\section{Resonant line scattering}

As discussed previously (e.g. Gil'fanov et al. 1987; Tawara et al. 1997; Shigeyama 1998) resonant scattering of line emission should be important in dense cores of galaxy clusters. This effect might well be the cause of the apparant abundance decrease towards the cluster center. To illustrate the effect we show in Fig. 13 the results of simple model calculations of the optical depth of some important resonance lines for an isothermal plasma halo with a temperature of $1.3 \mathrm{keV}$ and abundances as found for the radial range 1 to 1.5 arcmin. The main difference to previous model calculations (e.g. Shigeyama 1998) is the very small core radius $\left(20^{\prime \prime}, 1.97 \mathrm{kpc}\right)$ used here as indicated by the ROSAT HRI observations (e.g. Böhringer 1999). We note that all the $\mathrm{Ly} \alpha$ lines of the $\mathrm{H}$-like ions, the resonant lines

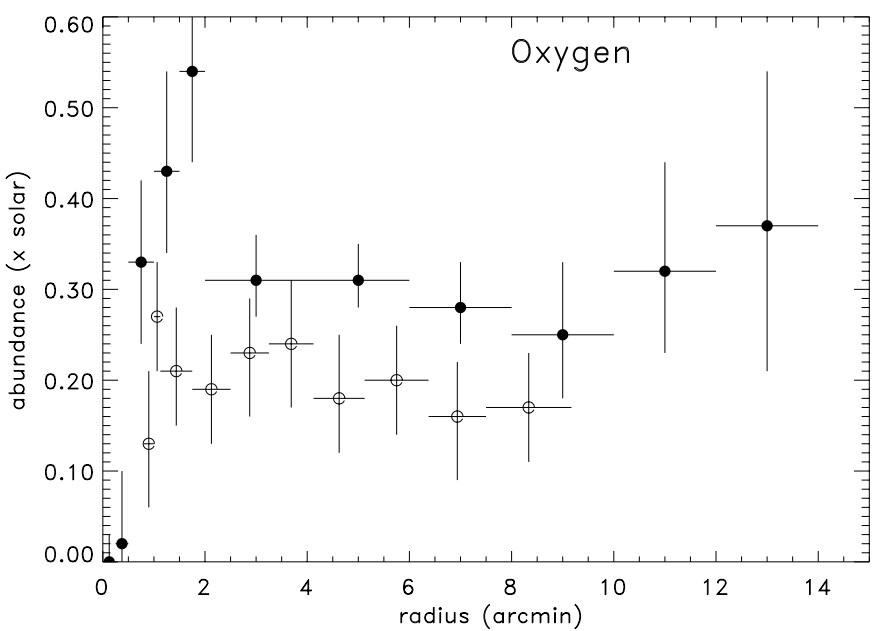

Fig. 12. Abundance profile of oxygen determined from MOS1 (open circles) and pn spectra (solid circles) in concentric rings around the M 87 nucleus excluding the regions of the X-ray lobes

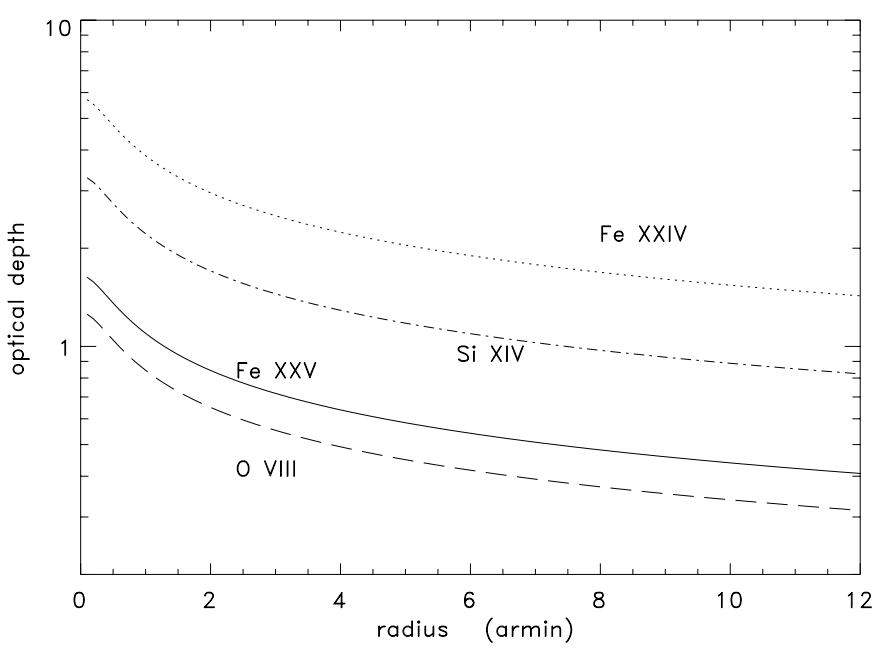

Fig. 13. Optical depth for resonant line scattering of some prominent emission lines of $\mathrm{Fe}, \mathrm{Si}$, and $\mathrm{O}$ observed in the $\mathrm{M} 87$ spectrum. For the plasma distribution we used a beta model with the parameters: core radius $=1.97 \mathrm{kpc}, \beta=0.47$, central electron density $=0.35 \mathrm{~cm}^{-3}$, and an isothermal temperature of $1.3 \mathrm{keV}$. The lines are labled by the relevant ions and refer to Fe XXV (6.69 keV), Fe XXIV (1.16 keV), Si XIV (2.003 $\mathrm{keV}$, Si Ly $\alpha)$, O VIII (0.652 keV, O Ly $\alpha)$

of the He-like ions and, many important Fe L-shell lines become moderately optically thick at the center. Since the optical thickness is moderate in the center and the density profile is quite steep, most of the last scattering must occur at a few core radii $\left(r=1^{\prime}\right.$ to $\left.2^{\prime}\right)$. In the most extreme case the effect will result in a flat surface brightness profile of the lines inside this radius. Since the abundances reflect the ratio of the line emission to the continuum emission and since the continuum surface brightness increases by a factor of about 8-10 from a radius of 1 to $1.5 \mathrm{arcmin}$ to the center, we expect at most a drop in the apparent abundances by the same factor. Therefore it seems that the results are very plausibly explained by resonant line 

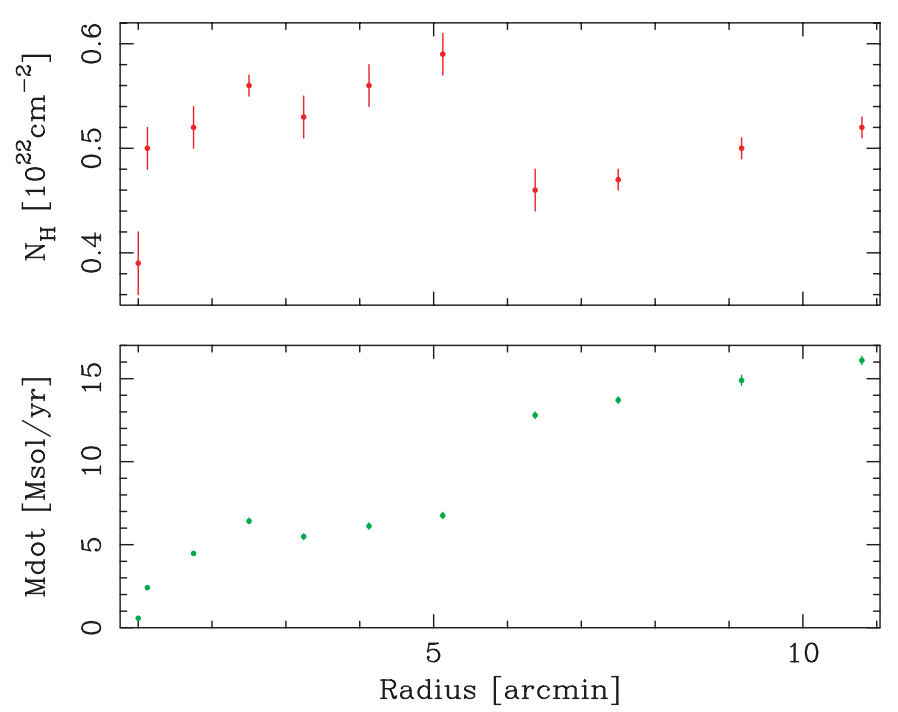

Fig. 14. Results of a cooling flow analysis in concentric circles around M 87 (using MOS1). Shown are the parameters for the warm absorption hydrogen column density in the cooling flow and the infered mass flow rate (for details see text)

scattering. Further modeling and a careful investigation of the X-ray spectra is necessary to provide a more detailed proof.

\section{Evidence for multi-temperature structure}

The spectral fitting results for concentric rings described above did not show any improvement by including more than one temperature component, except for the rings inside about 1-1.5. This is in contrast to current cooling flow models for which we would expect to see a multiphase medium out to a larger radius and a larger temperature range for the inner region. To further test the consistency with cooling flow models we performed combined model fits of a single temperature and cooling flow model (vmekal and mkcflow in XSPEC) for a set of circular regions around the M 87 center. Figure 14 shows the results for the two cooling flow parameters, mass deposition rate and internal absorption column density, as a function of the outer radius of the circular region. We expect to observe a decreasing absorption column with radius for the cooling flow model, we observe an increase, however. If we set the internal absorption to zero in the fit, the mass deposition rate is reduced to a value below $1 M_{\odot}$ per year. We interpret these results as inconsistent with the conventional cooling flow model, and the increasing mass deposition rate observed in Fig. 14 is forced onto the fit, because spatially separated temperatures in different rings are observed simultaneously in the spectra extracted from circular regions.

\section{Discussion and conclusions}

In Fig. 7 the spectral energy distribution of the bright knots in the jet are compared with a model for synchrotron radiation (from an electron spectrum of slope 2.3, with $\gamma_{\min }=100$ and $\gamma_{\max }=210^{6}$ in the equipartition magnetic field of $265 \mu$ Gauss). Also shown is the contribution of self-Comptonization for an equipartition field. Both components fall short of explaining the flux observed with $X M M$-Newton. The observations could be matched by synchrotron emission from an electron spectrum which extends to higher energies above a break at $\gamma \sim 810^{5}$, but the required steepening of the electron spectral slope of $\sim 1.5$ is not naturally predicted by simple models. The high electron energies and consequently short lifetimes imply that the electrons are accelerated in-situ at the X-ray knot (see also Heinz \& Begelman 1997). Lifetime effects could then explain why the optical and X-ray knots appear in slightly different positions.

The good agreement of the temperature profiles between the different $X M M$-Newton instruments as well as the ASCA and BeppoSAX instruments is a sign that the overall calibration of the detector response is already very good.

One of the surprising results is the non-detection of good evidence for a multi-temperature cooling flow structure for which M 87 was expected to be a good target. We also do not find the signature of oxygen absorption claimed to be seen by Buote (2000) in ROSAT PSPC data.

A striking result is the sharp drop of all deduced metal abundances at radii smaller than about 1 arcmin. This effect can be observed directly in Fig. 8. We have shown that resonant line scattering is important and could be responsible for this effect. This effect has also to be taken into account in the interpretation of XMM-RGS spectra of nearby clusters, since some of the relevant emission lines may suffer from scattering out-off the field-of-view of the spectrometer.

The decrease of the abundance profiles in the outer regions show a significant trend with element mass. The decrease from $r=1.5^{\prime}$ to $r=10^{\prime}$ is about a factor of 2 or larger for $\mathrm{Fe}, \mathrm{S}, \mathrm{Ar}$, less for Si (a factor of $\sim 1.7$ ), and the profile is almost constant for $\mathrm{O}$. This can be explained by a contribution to a homogeneous distribution by SN type II, the main source for O, and a SN type Ia yield of primarily heavy elements which is more concentrated in the center of the M 87 halo.

Acknowledgements. We thank the XMM software team for providing the Software Analysis System (SAS) for the $X M M$ Newton data reduction. H. B. thanks D. Grupe, F. Haberl for help with the SAS data analysis and E. M. Churazov for helpful comments. We are grateful to Martin Hardcastle for model-fitting software used to produce the model shown in Fig. 7. The paper is based on observations obtained with $X M M-N e w t o n$, an ESA science mission with instruments and contributions directly funded by ESA Member States and the USA (NASA). The XMM-Newton project is supported by the Bundesministerium für Bildung und Forschung, Deutsches Zentrum für Luft und Raumfahrt (BMBF/DLR), the MaxPlanck Society and the Haidenhain-Stiftung. 


\section{References}

Belsole, E., Sauvageot, J. L., Böhringer, H., et al. 2001, A\&A, 365, L188

Binggeli, B., Tammann, G. A., \& Sandage, A. 1987, AJ, 94, 251

Biretta, J. A., Stern, C. P., \& Harris, D. E. 1991, AJ, 101, 1632

Böhringer, H. 1999, in Diffuse Thermal and Relativistic Plasma in Galaxy Clusters, ed. H. Böhringer, L. Feretti, \& P. Schuecker, MPE Report, 115

Bolton, J. G., \& Stanley, G. S. 1948, Nat, 161, 312

Buote, D. A. 2000, ApJ, (in press), [astro-ph/0008408]

Byram, E. T., Chupp, T. A. A., \& Friedman, H. 1966, Sci, 152, 66

Canizares, C. R., Clark, G. W., Markert, T. H., et al. 1979, ApJ, 234, L33

Canizares, C. R., Clark, G. W., Jernigan, J. G., \& Markert, T. H. 1982, ApJ, 262, L32

Fabian, A. C., Nulsen, P. E. J., \& Canizares, C. R. 1984, Nat, 310,733

Freedman, W. L., Madore, B. F., Mould, J. R., et al. 1994, Nat, 371,757

Gil'fanov, M. R., Sunyaev, R. A., \& Churazov, E. M. 1987, Sov. Astron. Lett., 13, 3 (Pis'ma Astron. Zh. 13, 7, 1987)

Guainazzi, M., \& Molendi, S. 2000, A\&A, 351, L19

Heinz, S., \& Begelman, M. C. 1997, ApJ, 490, 653

Jansen, F., Lumb, D., Altieri, B., et al. 2001, A\&A, 365, L1

Mason, K. O., Breeveld, A., Much, R., et al. 2001, A\&A, 365, L36

Matsumoto, H., Koyama, K., Awaki, H., et al. 1996, PASJ, 48, 201

Meisenheimer, K., Roeser, H., \& Schloetelburg, M. 1996, A\&A, 307,61
Mushotzky, R. F., \& Szymkowiak, A. E. 1988, in Cooliong Flows in Clusters of Galaxies, ed. A. C. Fabian (Kluwer Acad. Publ.), Math. Phys. Sciences Ser., 229, 53

Neumann, M., Meisenheimer, K., Röser, H.-J., \& Fink, H. H. 1997, A\&A, 318, 383

Owen, F. N., Eilek, J. A., \& Keel, W. C. 1990, ApJ, 362, 449

Owen, F. N., Hardee, P. E., \& Cornwell, T. J. 1989, ApJ, 340, 698

Perola, G. C., \& Tarenghi, M. 1980, ApJ, 240, 447

Röser, H.-J., \& Meisenheimer, K. (eds.) 1999, The Radio Galaxy Messier 87, Proceedings of a workshop at Schloss Ringberg, 1997, Lecture notes in physics vol. 530 (Springer, Heidelberg)

Sakelliou, et al. 2000, A\&A, to be submitted

Schreier, E. J., Gorenstein, P., \& Feigelson, E. D. 1982, ApJ, 261,42

Shigeyama, T. 1998, ApJ, 497, 587

Sparks, W. B., Biretta, J. A., \& Macchetto, F. 1996, ApJ, 473, 254

Stewart, G. C., Canizares, C. R., Fabian, A. C., \& Nulsen, P. E. J. 1984, ApJ, 278, 536

Stocke, J. T., Lebofsky, M. J., \& Rieke, G. H. 1981, Nat, 294, 319

Strüder, L., Briel, U., Dennerl, K., et al. 2001, A\&A, 365, L18

Tammann, G. A., \& Federspiel, M. 1997, in Extragalactic Distance Scale, STScI Symposium, ed. M. Livio, et al. (Cambridge University Press), 137

Tansley, D. 2000, Ph.D. Thesis, University of Bristol

Tawara, Y., Akimoto, F., Kumada, A., Furuzawa, A., \& Yamashita, K. 1997, in X-ray Imaging and Spectroscopy of Cosmic Hot Plasmas, ed. F. Makino, \& K. Mitsuda (Universal Academy Press, Tokyo), 87

Turner, M. J. L., Abbey, A., Arnaud, M., et al. 2001, A\&A, $365, \mathrm{~L} 27$ 